\title{
On the number of coprime integer pairs within a circle
}

\author{
by
}

\author{
Wenguang Zhai (Jinan) and Xiaodong Cao (Beijing)
}

1. Introduction. Let $P(x)$ denote the number of integer pairs within the circle $a^{2}+b^{2} \leq x$, and $E(x)$ denote the difference $P(x)-\pi x$. Then the well-known circle problem is to estimate the upper bound of $E(x)$ and the best result at present is

$$
E(x)=O\left(x^{23 / 73+\varepsilon}\right) .
$$

See Huxley [6].

Let $V(x)$ denote the number of coprime integer pairs within the circle $a^{2}+b^{2} \leq x$. It is an exercise to deduce that

$$
V(x)=\frac{6}{\pi} x+O\left(x^{1 / 2} \exp \left(-c \log ^{3 / 5} x(\log \log x)^{-2 / 5}\right)\right),
$$

where $c$ is some absolute constant. The problem of reducing the exponent $1 / 2$ is open. One way to make progress is to assume the Riemann Hypothesis (RH). W. G. Nowak [11] proved that RH implies

$$
V(x)=\frac{6}{\pi} x+O\left(x^{15 / 38+\varepsilon}\right) .
$$

D. Hensley [5] also got a result of this type, but with a larger exponent.

The aim of this paper is to further improve this result. We have the following

THEOREM. If $R H$ is true, then

$$
V(x)=\frac{6}{\pi} x+O\left(x^{11 / 30+\varepsilon}\right) .
$$

Notations. $e(x)=\exp (2 \pi i x) \cdot m \sim M$ means $c_{1} M \leq m \leq c_{2} M$ for absolute constants $c_{1}$ and $c_{2}$. $E(x)$ always denotes the error term in the circle problem. $\varepsilon$ denotes an arbitrary small positive number and may be different at each occurrence.

1991 Mathematics Subject Classification: 11N37, 11P21.

This work is supported by Natural Science Foundation of Shandong Province (Grant No. Q98A02110). 
The authors thank Professor W. G. Nowak for kindly sending reprints of some of his papers.

2. Some preliminary lemmas and results. The following lemmas are needed.

Lemma 1. Suppose $0<c_{1} \lambda_{1} \leq\left|f^{\prime}(n)\right| \leq c_{2} \lambda_{1}$ and $\left|f^{\prime \prime}(n)\right| \sim \lambda_{1} N^{-1}$ for $N \leq n \leq c N$. Then

$$
\sum_{N<n \leq c N} e(f(n)) \ll \lambda_{1}^{-1}+\lambda_{1}^{1 / 2} N^{1 / 2} .
$$

Proof. If $c_{2} \lambda_{1} \leq 1 / 2$, this estimate is contained in the Kuz'min-Landau inequality; otherwise, the estimate follows from the well-known van der Corput's estimate for the second order derivative. Then

Lemma 2. Suppose $a(n)=O(1), 0<L \leq M<N \leq c L, L \gg 1, T \geq 2$.

$$
\begin{aligned}
\sum_{M<n \leq N} a(n)= & \frac{1}{2 \pi i} \int_{-T}^{T} \sum_{L<l \leq c L} \frac{a(l)}{l^{i t}} \cdot \frac{N^{i t}-M^{i t}}{t} d t \\
& +O\left(\min \left(1, \frac{L}{T\|M\|}\right)+\min \left(1, \frac{L}{T\|N\|}\right)\right. \\
& \left.+O\left(\frac{L \log (1+L)}{T}\right)\right) .
\end{aligned}
$$

Proof. This is the well-known Perron formula.

Lemma 3. Suppose $f(n) \ll P$ and $f^{\prime}(n) \gg \Delta$ for $n \sim N$. Then

$$
\sum_{n \sim N} \min \left(D, \frac{1}{\|f(n)\|}\right) \ll(P+1)\left(D+\Delta^{-1}\right) \log \left(2+\Delta^{-1}\right) .
$$

Pro of. This is contained in Lemma 2.8 of Krätzel [9].

Lemma 4. Suppose $a(n)$ are any complex numbers and $1 \leq Q \leq N$. Then

$$
\left|\sum_{N<n \leq c N} a(n)\right|^{2} \ll \frac{N}{Q} \sum_{0 \leq q \leq Q}\left(1-\frac{q}{Q}\right) \Re \sum_{N<n \leq c N-q} a(n) \overline{a(n+q)} .
$$

Proof. This is Weyl's inequality.

Lemma 5. Let $M \leq N<N_{1} \leq M_{1}$ and $a(n)$ be any complex numbers. Then

$$
\left|\sum_{N<n \leq N_{1}} a(n)\right| \leq \int_{-\infty}^{\infty} K(\theta)\left|\sum_{M<n \leq M_{1}} a(m) e(m \theta)\right| d \theta
$$


with $K(\theta)=\min \left(M_{1}-M+1,(\pi|\theta|)^{-1},(\pi|\theta|)^{-2}\right)$ and

$$
\int_{-\infty}^{\infty} K(\theta) d \theta \leq 3 \log \left(2+M_{1}-M\right)
$$

Proof. This is Lemma 2.2 of Bombieri and Iwaniec [2].

Lemma 6. Let $\alpha \beta \neq 0, \Delta>0, M \geq 1, N \geq 1$. Let $\mathcal{A}(M, N, \Delta)$ be the number of quadruples $(m, \widetilde{m}, n, \widetilde{n})$ such that

$$
\left|(\widetilde{m} / m)^{\alpha}-(\widetilde{n} / n)^{\beta}\right|<\Delta
$$

with $M \leq m, \widetilde{m} \leq 2 M$ and $N \leq n, \widetilde{n} \leq 2 N$. Then

$$
\mathcal{A}(M, N, \Delta) \ll M N \log 2 M N+\Delta M^{2} N^{2} .
$$

Proof. This is Lemma 1 of [3].

Lemma 7. Suppose $f(x)$ and $g(x)$ are algebraic functions in $[a, b]$ and

$$
\begin{aligned}
& \left|f^{\prime \prime}(x)\right| \sim \frac{1}{R}, \quad\left|f^{\prime \prime \prime}(x)\right| \ll \frac{1}{R U}, \\
& |g(x)| \ll G, \quad\left|g^{\prime}(x)\right| \ll G U_{1}^{-1}, \quad U, U_{1} \geq 1 .
\end{aligned}
$$

Then

$$
\begin{aligned}
\sum_{a<n \leq b} g(n) e(f(n))= & \sum_{\alpha<u \leq \beta} b_{u} \frac{g\left(n_{u}\right)}{\sqrt{\left|f^{\prime \prime}\left(n_{u}\right)\right|}} e\left(f\left(n_{u}\right)-u n_{u}+1 / 8\right) \\
& +O\left(G \log (\beta-\alpha+2)+G(b-a+R)\left(U^{-1}+U_{1}^{-1}\right)\right) \\
& +O(G \min (\sqrt{R}, 1 /\langle\alpha\rangle)+G \min (\sqrt{R}, 1 /\langle\beta\rangle))
\end{aligned}
$$

where $[\alpha, \beta]$ is the image of $[a, b]$ under the mapping $y=f^{\prime}(x), n_{u}$ is the solution of the equation $f^{\prime}(x)=u$,

$$
b_{u}= \begin{cases}1 & \text { for } \alpha<u<\beta \\ \frac{1}{2} & \text { for } u=\alpha \in \mathbb{Z} \text { or } u=\beta \in \mathbb{Z}\end{cases}
$$

and the function $\langle t\rangle$ is defined as follows:

$$
\langle t\rangle= \begin{cases}\|t\| & \text { if } t \text { is not an integer }, \\ \beta-\alpha & \text { otherwise, }\end{cases}
$$

where $\|t\|=\min _{n \in \mathbb{Z}}\{|t-n|\}$.

Proof. This is Theorem 2.2 of Min [10].

Lemma 8. Suppose $A_{i}, B_{j}, a_{i}$ and $b_{j}$ are all positive numbers. If $Q_{1}$ and $Q_{2}$ are real with $0<Q_{1} \leq Q_{2}$, then there exists some $q$ such that $Q_{1} \leq q \leq Q_{2}$ and 


$$
\begin{aligned}
\sum_{i=1}^{m} A_{i} q^{a_{i}}+\sum_{j=1}^{n} B_{j} q^{-b_{j}} & \\
& \leq 2^{m+n}\left(\sum_{i=1}^{m} \sum_{j=1}^{n}\left(A_{i}^{b_{j}} B_{j}^{a_{i}}\right)^{1 /\left(a_{i}+b_{j}\right)}+\sum_{i=1}^{m} A_{i} Q_{1}^{a_{i}}+\sum_{j=1}^{n} B_{j} Q_{2}^{-b_{j}}\right) .
\end{aligned}
$$

Proof. This is Lemma 3 of Srinivasan [12].

Lemma 9. Suppose $x$ is a large positive real number, $M, N, U$ are positive integers such that $1 \leq N \leq M^{1 / 4}, x^{\varepsilon} \leq M \leq x^{4 / 15}$ and $M^{1 / 6} \leq U \leq M^{5 / 6}$. Then

$$
\begin{aligned}
S & =\sum_{n \sim N} a_{n} \sum_{u \sim U} b_{u} \sum_{v \sim M U^{-1}} c_{v} e\left(\frac{\sqrt{n x}}{u v}\right) \\
& \ll\left(x^{1 / 12} M^{7 / 12} N^{11 / 12}+M^{11 / 12} N^{1 / 2}\right) \log x,
\end{aligned}
$$

where $a_{n}, b_{u}, c_{v}$ are coefficients satisfying $\left|a_{n}\right| \leq 1,\left|b_{u}\right| \leq 1,\left|c_{v}\right| \leq 1$.

Proof. We use Heath-Brown's method [4].

Without loss of generality, suppose $M^{1 / 2} \leq U \leq M^{5 / 6}$; otherwise we change the order of $U$ and $V=M U^{-1}$. Suppose $1 \leq Q \leq V N$ is a parameter to be determined. For each $q(1 \leq q \leq V N)$ define

$$
w_{q}=\left\{(v, n) \mid v \sim V, n \sim N, \frac{2 \sqrt{N}(q-1)}{V Q}<\frac{\sqrt{n}}{v}<\frac{2 \sqrt{N} q}{V Q}\right\} .
$$

Then

$$
S=\sum_{u \sim U} b_{u} \sum_{q=1}^{Q}\left(\sum_{(v, n) \in w_{q}} a_{n} c_{v} e\left(\frac{\sqrt{n x}}{u v}\right)\right) .
$$

By Cauchy's inequality

$$
\begin{aligned}
&|S|^{2} \ll U Q \sum_{u \sim U} \sum_{q=1}^{Q} \sum_{\left(v_{1}, n_{1}\right),\left(v_{2}, n_{2}\right) \in w_{q}} a_{n_{1}} \bar{a}_{n_{2}} \\
& \quad \times c_{v_{1}} \bar{c}_{v_{2}} e\left(\frac{\sqrt{x}}{u}\left(\frac{\sqrt{n_{1}}}{v_{1}}-\frac{\sqrt{n_{2}}}{v_{2}}\right)\right) \\
& \ll U Q \sum_{(*)}\left|\sum_{u \sim U} e\left(\frac{\sqrt{x}}{u}\left(\frac{\sqrt{n_{1}}}{v_{1}}-\frac{\sqrt{n_{2}}}{v_{2}}\right)\right)\right|,
\end{aligned}
$$

where $(*)$ denotes the condition

$$
\left|\frac{\sqrt{n_{1}}}{v_{1}}-\frac{\sqrt{n_{2}}}{v_{2}}\right| \leq \frac{2 \sqrt{N}}{V Q}, \quad v_{1}, v_{2} \sim V, n_{1}, n_{2} \sim N .
$$


Let $\lambda=\sqrt{n_{1}} / v_{1}-\sqrt{n_{2}} / v_{2}$. Then by Lemma 1 , the inner sum in (2.2) can be estimated by

$$
\min \left(U, \frac{U^{2}}{\sqrt{x}|\lambda|}\right)+x^{1 / 4}|\lambda|^{1 / 2} U^{-1 / 2} .
$$

By Lemma 6 , the contribution of $U$ (namely $|\lambda| \leq U x^{-1 / 2}$ ) to $|S|^{2}$ is

$$
U^{2} Q\left(V N \log 2 V N+U x^{-1 / 2} N^{3 / 2} V^{3}\right) \ll U^{2} Q V N \log 2 V N .
$$

The contribution of $U^{2} x^{-1 / 2}|\lambda|^{-1}\left(|\lambda|>U x^{-1 / 2}\right)$ is

$$
U^{2} Q V N(\log 2 V N)^{2}
$$

by a similar argument. The contribution of $x^{1 / 4}|\lambda|^{1 / 2} U^{-1 / 2}$ is (note $|\lambda| \ll$ $\sqrt{N} /(V Q), Q \ll V N)$

$$
x^{1 / 4} U^{1 / 2} N^{9 / 4} V^{3 / 2} Q^{-1 / 2} .
$$

Now, taking $Q=1+\left[x^{1 / 6} U^{-1} N^{5 / 6} V^{1 / 3}\right]$, we get

$$
|S| \log ^{-1} 2 V N \ll U V^{1 / 2} N^{1 / 2}+x^{1 / 12} U^{1 / 2} V^{2 / 3} N^{11 / 12},
$$

whence the lemma follows since $M^{1 / 2} \leq U \leq M^{5 / 6}$.

Lemma 10. Suppose $x, M, N$ satisfy the conditions of Lemma 9. Then

$$
\begin{aligned}
T(M, N) & =\sum_{n \sim N} a(n) \sum_{m \sim M} \mu(m) e\left(\frac{\sqrt{n x}}{m}\right) \\
& \ll\left(x^{1 / 12} M^{7 / 12} N^{11 / 12}+M^{11 / 12} N^{1 / 2}\right) x^{\varepsilon},
\end{aligned}
$$

where $|a(n)| \leq 1$.

Proof. By Heath-Brown's identity $(k=4), T(M, N)$ can be written as $O\left(\log ^{8} x\right)$ sums of the form

$$
T=\sum_{n \sim N} a(n) \sum_{m_{1} \sim M_{1}} \ldots \sum_{m_{8} \sim M_{8}} \mu\left(m_{1}\right) \ldots \mu\left(m_{4}\right) e\left(\frac{\sqrt{n x}}{m_{1} \ldots m_{8}}\right),
$$

where $M \ll M_{1} \ldots M_{8} \ll M, M_{1}, M_{2}, M_{3}, M_{4} \leq(2 M)^{1 / 4}$. Some $m_{i}$ may only take value 1 .

To prove the lemma we consider three cases.

CASE 1: There is some $M_{i}$ such that $M_{i}>M^{5 / 6}$. It must follow that $i \geq 5 ; i=8$ for example. We use the exponent pair $(1 / 6,4 / 6)$ to estimate the sum on $m_{8}$ and estimate other variables trivially, to get $\left(F=\sqrt{N x} M^{-1}\right)$

$$
\begin{aligned}
T & \ll N M M_{8}^{-1}\left(\frac{M_{8}}{F}+\left(\frac{F}{M_{8}}\right)^{1 / 6} M_{8}^{2 / 3}\right) \\
& \ll x^{-1 / 2} M^{2} N^{1 / 2}+x^{1 / 12} M^{5 / 12} N^{13 / 12} \\
& \ll x^{1 / 12} M^{5 / 12} N^{13 / 12} \ll x^{1 / 12} M^{7 / 12} N^{11 / 12} .
\end{aligned}
$$


CASE 2: There is some $M_{i}$ satisfying $M^{1 / 6} \leq M_{i} \leq M^{5 / 6}$. Let $u=m_{i}$, $v=\prod_{j \neq i} m_{j}, U=M_{i}$. Then by Lemma 9 we get

$$
x^{-\varepsilon} T \ll x^{1 / 12} M^{7 / 12} N^{11 / 12}+M^{11 / 12} N^{1 / 2},
$$

where $x^{\varepsilon}$ comes from the divisor argument.

CASE 3: All $M_{i}$ satisfy $M_{i} \leq M^{1 / 6}$. Without loss of generality, suppose $M_{1} \geq M_{2} \geq \ldots \geq M_{8}$. Let $l$ be the first positive integer $j$ such that

$$
M_{1} \ldots M_{j}>M^{1 / 6}
$$

then $l \geq 2$. Thus we have

$$
M_{1} \ldots M_{l} \leq\left(M_{1} \ldots M_{l-1}\right) M_{l} \leq M^{2 / 6} .
$$

Now take $u=m_{1} \ldots m_{j}, v=m_{j+1} \ldots m_{6}, U=M_{1} \ldots M_{j}$. By Lemma 9, (2.10) still holds.

Lemma 10 follows from the three cases.

Now we prove the following proposition. It plays an important role in this paper. The idea of the proof has been used by several authors; see Jia [8], Baker and Harman [1], for example.

Proposition 1. Suppose $M, N$ are large positive numbers, $A>0, \alpha, \beta$ are rational numbers (not non-negative numbers). Suppose $m \sim M, n \sim N$, $F=A M^{\alpha} N^{\beta} \gg N,|a(m)| \leq 1,|b(n)| \leq 1$. Then

$$
\begin{aligned}
S= & \sum_{M<m \leq 2 M} \sum_{N<n \leq 2 N} a(m) b(n) e\left(A m^{\alpha} n^{\beta}\right) \\
\ll & \left(M N^{1 / 2}+F^{4 / 20} M^{13 / 20} N^{15 / 20}+F^{4 / 23} M^{15 / 23} N^{18 / 23}\right. \\
& \left.+F^{1 / 6} M^{2 / 3} N^{7 / 9}+F^{1 / 5} M^{3 / 5} N^{4 / 5}+F^{1 / 10} M^{4 / 5} N^{7 / 10}\right) \log ^{4} F .
\end{aligned}
$$

Proof. Without loss of generality, we suppose $\beta>0$; for $\beta<0$, the proof is the same. By Cauchy's inequality and Lemma 4 we get

$$
\begin{aligned}
|S|^{2} & \ll M \sum_{M<m \leq 2 M}\left|\sum_{N<n \leq 2 N} b(n) e\left(A m^{\alpha} n^{\beta}\right)\right|^{2} \\
& \ll \frac{M^{2} N^{2}}{Q}+\frac{M N}{Q}\left|\Sigma_{1}\right|
\end{aligned}
$$

with

$$
\Sigma_{1}=\sum_{q=1}^{Q}\left(1-\frac{q}{Q}\right) \sum_{N<n \leq 2 N-q} \overline{b(n)} b(n+q) \sum_{M<m \leq 2 M} e\left(A m^{\alpha} g(n, q)\right),
$$

where $Q$ is a parameter satisfying $\log N \leq Q \leq N \log ^{-1} N, g(n, q)=$ $(n+q)^{\beta}-n^{\beta}$. 
We write

$$
\begin{aligned}
\Sigma_{1}= & \sum_{1 \leq q \leq Q} \sum_{2 N-Q<n \leq 2 N-q} \sum_{m}+\sum_{q \leq B} \sum_{N<n \leq 2 N-Q} \sum_{m} \\
& +\sum_{B \leq q \leq Q} \sum_{N<n \leq 2 N-Q} \sum_{m}=\Sigma_{2}+\Sigma_{3}+\Sigma_{4},
\end{aligned}
$$

where $B=\max (\log N, M N /(c(\alpha, \beta)) F)$ and

$$
c(\alpha, \beta)=2|\alpha \beta| \max \left(2^{\alpha-1}, 1\right) \max \left(2^{\beta-1}, 1\right) .
$$

By Lemma 1 we have

$$
\begin{aligned}
& \Sigma_{2} \ll\left(\frac{M N Q}{F}+\frac{F^{1 / 2} Q^{5 / 2}}{N^{1 / 2}}\right) \log N, \\
& \Sigma_{3} \ll\left(\frac{M N^{2}}{F}+F^{1 / 2} N^{1 / 2}\right) \log ^{2} N .
\end{aligned}
$$

So we only need to bound $\Sigma_{4}$. Notice that $\Sigma_{4}$ can be written as the sum of $O(\log Q)$ exponential sums of the form

$$
\Sigma_{5}=\sum_{Q_{1}<q \leq 2 Q_{1}} c(q) \sum_{N<n \leq 2 N-Q} \overline{b(n)} b(n+q) \sum_{M<m \leq 2 M} e\left(A m^{\alpha} g(n, q)\right),
$$

where $B \leq Q_{1} \leq Q / 2, c(q)=1-q / Q$.

By Lemma 7 we have

$$
\begin{aligned}
& \sum_{M<m \leq 2 M} e\left(A m^{\alpha} g(n, q)\right) \\
& =c_{3} \sum_{U_{1}<u \leq U_{2}} \frac{(A g)^{1 /(2(1-\alpha))}}{u^{(1-\alpha / 2) /(1-\alpha)}} e\left(c_{4} A^{1 /(1-\alpha)} g^{1 /(1-\alpha)} u^{-\alpha /(1-\alpha)}\right) \\
& \quad+O\left(\log F+\frac{M N}{F q}+\min \left(\frac{M N^{1 / 2}}{F^{1 / 2} q^{1 / 2}}, \max \left(\frac{1}{\left\langle U_{2}\right\rangle}, \frac{1}{\left\langle U_{1}\right\rangle}\right)\right)\right),
\end{aligned}
$$

where $U_{1}=c_{5} A M^{\alpha-1} g, U_{2}=c_{6} A M^{\alpha-1} g, g=g(n, q)$. By Lemma 3 , the contribution of the error term to $\Sigma_{5}$ is

$$
N Q_{1} \log F+M N^{2} F^{-1} \log Q_{1}+F^{1 / 2} Q_{1}^{3 / 2} N^{-1 / 2} .
$$

It can be easily seen that $g(n, q)<\beta q n^{\beta-1}$ for $0<\beta<1, g(n, q)>$ $\beta q n^{\beta-1}$ for $\beta>1$. If $\beta q A M^{\alpha-1} n^{\beta-1}-A M^{\alpha-1} g>\log ^{-1} N$, then by the trivial estimate we have $(i=5,6)$

$$
\begin{aligned}
& \sum_{q \sim q_{1}} \sum_{n \sim N} \mid \sum_{c_{i} A M^{\alpha-1}} \sum_{g<u \leq c_{i} \beta q A M^{\alpha-1} n^{\beta-1}} \frac{(A g)^{1 /(2(1-\alpha))}}{u^{(1-\alpha / 2) /(1-\alpha)}} \\
& \times e\left(c_{4} A^{1 /(1-\alpha)} g^{1 /(1-\alpha)} u^{-\alpha /(1-\alpha)}\right) \mid \\
& \ll F^{1 / 2} Q_{1}^{5 / 2} N^{-1 / 2} \log ^{2} N .
\end{aligned}
$$


Now suppose $\beta q A M^{\alpha-1} n^{\beta-1}-A M^{\alpha-1} g \leq \log ^{-1} N$. Notice that the fact that

$$
\left[c_{i} \beta q A M^{\alpha-1} n^{\beta-1}\right]-\left[c_{i} A M^{\alpha-1} g\right]=1
$$

always implies

$$
\left\|c_{i} A M^{\alpha-1} g\right\| \leq c_{i} \beta q A M^{\alpha-1} n^{\beta-1}-c_{i} A M^{\alpha-1} g=\delta_{i} .
$$

We have

$$
\begin{aligned}
\sum_{c_{i} A M^{\alpha-1} g<u \leq c_{i} \beta q A M^{\alpha-1} n^{\beta-1}} & \frac{(A g)^{1 /(2(1-\alpha))}}{u^{(1-\alpha / 2) /(1-\alpha)}} \\
& \ll \frac{M N^{1 / 2}}{F^{1 / 2} Q_{1}^{1 / 2}} \sum_{c_{i} A M^{\alpha-1} g<u \leq c_{i} \beta q A M^{\alpha-1} n^{\beta-1}} \\
& \ll \frac{M N^{1 / 2}}{F^{1 / 2} Q_{1}^{1 / 2}} \min \left(1, \frac{\delta_{i}}{\left\|c_{i} A M^{\alpha-1} g\right\|}\right) \\
& \ll \frac{M N^{1 / 2}}{F^{1 / 2} Q_{1}^{1 / 2}} \min \left(1, \frac{F Q_{1}^{2}}{M N^{2}} \cdot \frac{1}{\left\|c_{i} A M^{\alpha-1} g\right\|}\right),
\end{aligned}
$$

thus by Lemma 3 ,

$$
\begin{aligned}
\sum_{q \sim q_{1}} \sum_{n \sim N} \mid \sum_{c_{i} A M^{\alpha-1}} \sum_{g<u \leq c_{i} \beta q A M^{\alpha-1} n^{\beta-1}} \frac{(A g)^{1 /(2(1-\alpha))}}{u^{(1-\alpha / 2) /(1-\alpha)}} \\
\times e\left(c_{4} A^{1 /(1-\alpha)} g^{1 /(1-\alpha)} u^{-\alpha /(1-\alpha)}\right) \mid \\
\ll F^{1 / 2} Q_{1}^{3 / 2} N^{-1 / 2} \log ^{2} N .
\end{aligned}
$$

Now it suffices to bound

$$
\begin{aligned}
\Sigma_{6}= & \sum_{Q_{1}<q \leq 2 Q_{1}} c(q) \sum_{N<n \leq 2 N-Q} \overline{b(n)} b(n+q) \\
& \times \sum_{u} \frac{(A g)^{1 /(2(1-\alpha))}}{u^{(1-\alpha / 2) /(1-\alpha)}} e\left(c_{4} A^{1 /(1-\alpha)} g^{1 /(1-\alpha)} u^{-\alpha /(1-\alpha)}\right),
\end{aligned}
$$

where

$$
c_{5} \beta q A M^{\alpha-1} n^{\beta-1}<u \leq c_{6} \beta q A M^{\alpha-1} n^{\beta-1} .
$$

By Lemmas 2 and 3 we get (choose $T=F^{10}$ )

$$
\Sigma_{6} \ll\left|\Sigma_{7}\right|+F^{1 / 2} Q_{1}^{3 / 2} N^{-1 / 2} \log N
$$

with 


$$
\begin{aligned}
\Sigma_{7}= & \sum_{Q_{1}<q \leq 2 Q_{1}} c(q) \sum_{N<n \leq 2 N-Q} \overline{b(n)} b(n+q) \sum_{u} \frac{(A g)^{1 /(2(1-\alpha))}}{u^{(1-\alpha / 2) /(1-\alpha)}} \\
& \times \frac{q^{i t} A^{i t} M^{(\alpha-1) i t} n^{(\beta-1) i t}}{u^{i t}} e\left(c_{4} A^{1 /(1-\alpha)} g^{1 /(1-\alpha)} u^{-\alpha /(1-\alpha)}\right),
\end{aligned}
$$

where $t$ is a real number independent of the variables and

$$
c_{7} F Q_{1}(M N)^{-1}<u \leq c_{8} F Q_{1}(M N)^{-1} .
$$

It is easy to see that

$$
\begin{aligned}
\Sigma_{7}= & \sum_{u} \frac{A^{1 /(2(1-\alpha))+i t} M^{(\alpha-1) i t}}{u^{(1-\alpha / 2) /(1-\alpha)+i t}} \sum_{N<n \leq 2 N-Q} \overline{b(n)} n^{(\beta-1) i t} \\
& \times \sum_{Q_{1}<q \leq 2 Q_{1}} j(q) b(n+q) g^{1 /(2(1-\alpha))} \\
& \times e\left(c_{4} A^{1 /(1-\alpha)} g^{1 /(1-\alpha)} u^{-\alpha /(1-\alpha)}\right) \\
\ll & \sum_{u} \frac{(A g)^{1 /(2(1-\alpha))}}{u^{(1-\alpha / 2) /(1-\alpha)}}\left(Q_{1} N^{\beta-1}\right)^{1 /(2(1-\alpha))} \Sigma_{8}(u) \\
\ll & F^{1 / 2} Q_{1}^{1 / 2} N^{-1 / 2} \Sigma_{8}\left(u_{0}\right)
\end{aligned}
$$

for some $u_{0}$ with

$$
\begin{aligned}
& \Sigma_{8}(u)=\sum_{N<n \leq 2 N-Q} \mid \sum_{Q_{1}<q \leq 2 Q_{1}} j(q) b(n+q) g_{0}^{1 /(2(1-\alpha))} \\
& \quad \times e\left(c_{4} A^{1 /(1-\alpha)} g^{1 /(1-\alpha)} u^{-\alpha /(1-\alpha)}\right) \mid,
\end{aligned}
$$

where $j(q)=c(q) q^{i t}, 1 \ll g_{0}=g\left(Q_{1} N^{\beta-1}\right)^{-1} \ll 1$.

Suppose $10 \leq R \leq Q_{1} \log ^{-1 / 2} N$ is a parameter to be determined. By Cauchy's inequality and Lemma 4 we get

$$
\begin{aligned}
\Sigma_{8}(u)^{2} \ll & N \sum_{n} \mid \sum_{q} j(q) b(n+q) g_{0}^{1 /(2(1-\alpha))} \\
& \times\left. e\left(c_{4} A^{1 /(1-\alpha)} g^{1 /(1-\alpha)} u^{-\alpha /(1-\alpha)}\right)\right|^{2} \\
\ll & \frac{N^{2} Q_{1}^{2}}{R}+\frac{N Q_{1}}{R} \sum_{1 \leq r \leq R}\left|E_{r}\right|,
\end{aligned}
$$

where

$$
\begin{aligned}
E_{r}= & \sum_{N<n \leq 2 N-Q} \sum_{Q_{1}<q \leq 2 Q_{1}-r} j(q) b(n+q) g_{0}^{1 /(2(1-\alpha))}(n, q) \\
& \times j(q+r) b(n+q+r) g_{0}^{1 /(2(1-\alpha))}(n, q+r) \\
& \times e\left(c_{4} A^{1 /(1-\alpha)} u^{-\alpha /(1-\alpha)}\left(g^{1 /(2(1-\alpha))}(n, q)-g^{1 /(2(1-\alpha))}(n, q+r)\right)\right) .
\end{aligned}
$$


So it reduces to bound $E_{r}$ for fixed $r$. Making the change of variable $n+q=l$, we have

$$
\begin{aligned}
E_{r}= & \sum_{Q_{1}<q \leq 2 Q_{1}-r} j(q) \overline{j(q+r)} \sum_{N+q<l \leq 2 N+q-Q} b(l) \overline{b(l+r)} g_{0}^{1 /(2(1-\alpha))}(l-q, q) \\
& \times g_{0}^{1 /(2(1-\alpha))}(l-q, q+r) \\
& \times e\left(c_{4} A^{1 /(1-\alpha)} u^{-\alpha /(1-\alpha)}\left(g^{1 /(2(1-\alpha))}(l, q)-g^{1 /(2(1-\alpha))}(l, q+r)\right)\right) \\
= & \sum^{N} \sum_{Q_{1}<n \leq 2 N+2 Q_{1}-r-Q} b(n) \overline{b(n+r)} \\
& \times \sum_{\max \left(Q_{1}, n-2 N+Q\right)<q \leq \min \left(2 Q_{1}-r, n-N\right)} j(q) \overline{j(q+r)} \\
& \times g_{0}^{1 /(2(1-\alpha))}(n-q, q) g_{0}^{1 /(2(1-\alpha))}(n-q, q+r) \\
& \times e\left(c_{4} A^{1 /(1-\alpha)} u^{-\alpha /(1-\alpha)} k(n, q, r)\right),
\end{aligned}
$$

where

$$
k(n, q, r)=g^{1 /(2(1-\alpha))}(n-q, q)-g^{1 /(2(1-\alpha))}(n-q, q+r) .
$$

By Lemma 5 we get

$$
\begin{aligned}
E_{r} \log ^{-1} N & \ll \sum_{n \sim N} \mid \sum_{q \sim Q_{1}} j(q) \overline{j(q+r)} g_{0}^{1 /(2(1-\alpha))}(n-q, q) \\
& \times g_{0}^{1 /(2(1-\alpha))}(n-q, q+r) e\left(c_{4} A^{1 /(1-\alpha)} u^{-\alpha /(1-\alpha)} k(n, q, r)+\theta_{0} q\right) \mid,
\end{aligned}
$$

where $\theta_{0}$ is a real number independent of $n$ and $q$.

Suppose $10 \leq T \leq Q_{1} \log ^{-1 / 2} N$ is a parameter to be determined. By Cauchy's inequality and Lemma 4 we get

$$
\left|E_{r}\right|^{2} \log ^{-2} N \ll \frac{N^{2} Q_{1}^{2}}{T}+\frac{N Q_{1}}{T}\left|D_{t}(r)\right|,
$$

with

$$
\begin{aligned}
D_{t}(r)= & \sum_{n \sim N} \sum_{Q_{1}<q \leq 2 Q_{1}-t} j(q) \overline{j(q+r)} g_{0}^{1 /(2(1-\alpha))}(n-q, q) \\
& \times g_{0}^{1 /(2(1-\alpha))}(n-q, q+r) j(q+t+r) \overline{j(q+t)} \\
& \times g_{0}^{1 /(2(1-\alpha))}(n-q-t, q+t) g_{0}^{1 /(2(1-\alpha))}(n-q-t, q+t+r) \\
& \times e\left(c_{4} A^{1 /(1-\alpha)} u^{-\alpha /(1-\alpha)}(k(n, q, r)-k(n, q+t, r))\right) \\
\ll & \sum_{q \sim Q_{1}}\left|\sum_{n \sim N} \phi(n) e(f(n))\right|,
\end{aligned}
$$


where

$$
\begin{aligned}
\phi(n)= & g_{0}^{1 /(2(1-\alpha))}(n-q, q) g_{0}^{1 /(2(1-\alpha))}(n-q, q+r) \\
& \times g_{0}^{1 /(2(1-\alpha))}(n-q-t, q+t) g_{0}^{1 /(2(1-\alpha))}(n-q-t, q+t+r)
\end{aligned}
$$

and

$$
f(n)=c_{4} A^{1 /(1-\alpha)} u^{-\alpha /(1-\alpha)}(k(n, q, r)-k(n, q+t, r)) .
$$

It is an easy exercise to verify that $\phi(n)$ is monotonic and

$$
\left|f^{\prime}(n)\right| \sim \frac{F r t}{Q_{1} N^{2}}, \quad\left|f^{\prime \prime}(n)\right| \sim \frac{F r t}{Q_{1} N^{3}} \quad(N<n \leq 2 N) ;
$$

thus by Lemma 1 we get

$$
D_{t}(r) \ll Q_{1}\left(\frac{Q_{1} N^{2}}{F r t}+\frac{(F r t)^{1 / 2}}{\left(Q_{1} N\right)^{1 / 2}}\right)=\frac{Q_{1}^{2} N^{2}}{F r t}+\frac{\left(F r t Q_{1}\right)^{1 / 2}}{N^{1 / 2}} .
$$

Inserting (2.28) into (2.27) we get

$$
\left|E_{r}\right|^{2} \log ^{-2} N \ll \frac{Q_{1}^{2} N^{2}}{T}+\frac{Q_{1}^{3} N^{3}}{F r T}+Q_{1}^{3 / 2}(N F T r)^{1 / 2} .
$$

Notice that $(2.29)$ is also true for $0<T<10$; then by Lemma 8 choosing a best $T \in\left(0, Q_{1} \log ^{-1 / 2} N\right)$ we get

$$
\left|E_{r}\right|^{2} \log ^{-2} N \ll N^{1 / 2} Q_{1}^{5 / 6} F^{1 / 6} r^{1 / 6}+N^{2 / 3} Q_{1}+N Q_{1}^{1 / 2}+\frac{N^{3 / 2} Q_{1}}{F^{1 / 2} r^{1 / 2}} .
$$

Inserting (2.30) into (2.26) we have

$$
\begin{aligned}
& \Sigma_{8}(u)^{2} \log ^{-2} N \\
& \ll \frac{Q_{1}^{2} N^{2}}{R}+N^{3 / 2} Q_{1}^{11 / 6} F^{1 / 6} R^{1 / 6}+\frac{N^{5 / 2} Q_{1}^{2}}{F^{1 / 2} R^{1 / 2}}+N^{5 / 3} Q_{1}^{2}+N^{2} Q_{1}^{3 / 2} .
\end{aligned}
$$

This is also true for $0<R \leq 10$. Choosing a best $R \in\left(0, Q_{1} \log ^{-1 / 2} N\right)$ via Lemma 8 we get

$$
\begin{aligned}
\Sigma_{8}(u) \log ^{-2} N \ll & N^{11 / 14} Q_{1}^{13 / 14} F^{1 / 14}+N^{14 / 16} Q_{1}^{15 / 16} \\
& +N^{5 / 4} Q_{1}^{3 / 4} F^{-1 / 4}+N^{5 / 6} Q_{1}+N Q_{1}^{3 / 4} .
\end{aligned}
$$

Inserting (2.32) into (2.24) we have

$$
\begin{aligned}
\Sigma_{7} \ll & N^{4 / 14} Q_{1}^{20 / 14} F^{8 / 14}+N^{6 / 16} Q_{1}^{23 / 16} F^{8 / 16}+N^{3 / 4} Q_{1}^{5 / 4} F^{1 / 4} \\
& +N^{1 / 3} Q_{1}^{3 / 2} F^{1 / 2}+N^{1 / 2} Q_{1}^{5 / 4} F^{1 / 2}
\end{aligned}
$$

Combining (2.17), (2.18), (2.20), (2.22) and (2.33) we get

$$
\begin{aligned}
\Sigma_{5} \log ^{-4} F \ll & N^{4 / 14} Q^{20 / 14} F^{8 / 14}+N^{6 / 16} Q^{23 / 16} F^{8 / 16} \\
& +N^{3 / 4} Q^{5 / 4} F^{1 / 4}+N^{1 / 3} Q^{3 / 2} F^{1 / 2} \\
& +N^{1 / 2} Q^{5 / 4} F^{1 / 2}+N Q+M N^{2} F^{-1} \\
& +F^{1 / 2} Q^{5 / 2} N^{-1 / 2}
\end{aligned}
$$


Inserting (2.14), (2.15) and (2.34) into (2.12) we get

$$
\begin{aligned}
|S|^{2} \log ^{-6} F \ll & \frac{M^{2} N^{2}}{Q}+M N^{18 / 14} Q^{6 / 14} F^{8 / 14} \\
& +M N^{22 / 16} Q^{7 / 16} F^{8 / 16}+M N^{7 / 4} Q^{1 / 4} F^{1 / 4} \\
& +M N^{4 / 3} Q^{1 / 2} F^{1 / 2}+M N^{3 / 2} Q^{1 / 4} F^{1 / 2}+M N^{2} \\
& +M^{2} N^{3} Q^{-1} F^{-1}+M N^{1 / 2} Q^{3 / 2} F^{1 / 2} .
\end{aligned}
$$

Since $Q<N \ll F$, we have

$$
\begin{aligned}
M N^{2}+M N^{7 / 4} Q^{1 / 4} F^{1 / 4} & \ll M N^{3 / 2} Q^{1 / 4} F^{1 / 2}, \\
M^{2} N^{3}(F Q)^{-1} & \ll M^{2} N^{2} Q^{-1} .
\end{aligned}
$$

So we obtain

$$
\begin{aligned}
|S|^{2} \log ^{-6} F \ll & \frac{M^{2} N^{2}}{Q}+M N^{18 / 14} Q^{6 / 14} F^{8 / 14} \\
& +M N^{22 / 16} Q^{7 / 16} F^{8 / 16}+M N^{4 / 3} Q^{1 / 2} F^{1 / 2} \\
& +M N^{3 / 2} Q^{1 / 4} F^{1 / 2}+M N^{1 / 2} Q^{3 / 2} F^{1 / 2}
\end{aligned}
$$

Note that (2.36) is trivial for $0<Q \leq \log N$. Now the proposition follows from choosing a best $Q \in\left(0, N \log ^{-1} N\right)$ via Lemma 8 .

3. An expression of the error term. In the rest of this paper, we always use $E_{P}(x)$ to denote the difference $V(x)-\frac{6}{\pi} x$. The aim of this section is to give an expression of $E_{P}(x)$ subject to $\mathrm{RH}$.

Let $y$ be a parameter, $x^{\varepsilon} \leq y \leq x^{1 / 2-\varepsilon}$,

$$
f_{1}(s)=\sum_{n \leq y} \mu(n) n^{-s}, \quad f_{2}(s)=\zeta^{-1}(s)-f_{1}(s) .
$$

Let $r(n)$ be the number of representations of $n$ as a sum of two squares. Then

$$
\begin{aligned}
V(x) & =\sum_{\substack{a^{2}+b^{2} \leq x \\
(a, b)=1}} 1=\sum_{a^{2}+b^{2} \leq x} \sum_{m \mid(a, b)} \mu(m) \\
& =\sum_{m^{2}\left(a^{2}+b^{2}\right) \leq x} \mu(m)=\sum_{m^{2} k \leq x} \mu(m) r(k) \\
& =\sum_{m \leq y}+\sum_{m>y}=\Sigma_{1}+\Sigma_{2}, \quad \text { say. }
\end{aligned}
$$

Notice that for $\sigma>1$,

$$
\sum_{n=1}^{\infty} r(n) n^{-s}=4 \zeta(s) L(s, \chi),
$$


where $\chi$ is the non-principal character $\bmod 4$, we have

$$
\begin{aligned}
\Sigma_{1} & =\sum_{m \leq y} \mu(m) P\left(\frac{x}{m^{2}}\right) \\
& =\sum_{m \leq y} \mu(m)\left(\operatorname{Res}_{s=1} \frac{4 \zeta(s) L(s, \chi)}{s}\left(\frac{x}{m^{2}}\right)^{s}+E\left(\frac{x}{m^{2}}\right)\right) \\
& =\operatorname{Res}_{s=1}\left(4 f_{1}(2 s) \zeta(s) L(s, \chi) x^{s} s^{-1}\right)+\sum_{m \leq y} \mu(m) E\left(\frac{x}{m^{2}}\right) .
\end{aligned}
$$

To treat $\Sigma_{2}$ we begin with

$$
f_{2}(s)=\sum_{m>y} \mu(m) m^{-s}
$$

for $\sigma>1$. Hence

$$
4 f_{2}(2 s) \zeta(s) L(s, \chi)=\sum_{n=1}^{\infty} b(n) n^{-s} \quad(\sigma>1),
$$

where

$$
b(n)=\sum_{n=m^{2} k, m>y} \mu(m) r(k) .
$$

By Perron's formula we have

$$
\Sigma_{2}=\sum_{n \leq x} b(n)=\frac{1}{2 \pi i} \int_{1+\varepsilon-i x^{2}}^{1+\varepsilon+i x^{2}} g(s) d s+O\left(x^{\varepsilon}\right),
$$

where

$$
g(s)=4 f_{2}(2 s) \zeta(s) L(s, \chi) x^{s} s^{-1} .
$$

By Cauchy's theorem, we have

$$
\frac{1}{2 \pi i} \int_{1+\varepsilon-i x^{2}}^{1+\varepsilon+i x^{2}} g(s) d s=I_{1}+I_{2}-I_{3}+\operatorname{Res}_{s=1} g(s)
$$

where

$$
\begin{gathered}
I_{1}=\frac{1}{2 \pi i} \int_{0.5+\varepsilon+i x^{2}}^{1+\varepsilon+i x^{2}} g(s) d s, \quad I_{2}=\frac{1}{2 \pi i} \int_{0.5+\varepsilon-i x^{2}}^{0.5+\varepsilon+i x^{2}} g(s) d s, \\
I_{3}=\frac{1}{2 \pi i} \int_{0.5+\varepsilon-i x^{2}}^{1+\varepsilon-i x^{2}} g(s) d s .
\end{gathered}
$$

Since RH is true, it follows that

$$
\zeta(s) \ll|t|^{\varepsilon}+1, \quad \sigma \geq 0.5+\varepsilon,
$$




$$
f_{2}(2 s) \ll y^{-1 / 2}\left(|t|^{\varepsilon}+1\right), \quad \sigma \geq 0.5+\varepsilon .
$$

For $L(s, \chi)$, we have

$$
L(s, \chi) \ll(|t|+1)^{(1-\sigma) / 2}, \quad 0.5 \leq \sigma \leq 1 .
$$

Using (3.8)-(3.10) we get

$$
\begin{gathered}
I_{1}-I_{3} \ll y^{-1 / 2}\left(1+x^{\varepsilon}\right), \\
I_{2} \ll y^{-1 / 2} x^{1 / 2+\varepsilon}\left(\int_{1}^{x^{2}} \frac{|L(1 / 2+\varepsilon+i t, \chi)|}{t} d t+1\right) \\
\ll y^{-1 / 2} x^{1 / 2+\varepsilon} .
\end{gathered}
$$

Combining (3.6)-(3.12) we get

$$
\Sigma_{2}=\operatorname{Res}_{s=1}\left(4 f_{2}(2 s) \zeta(s) L(s, \chi) x^{s} s^{-1}\right)+O\left(y^{-1 / 2} x^{1 / 2+\varepsilon}\right) .
$$

From (3.2), (3.4) and (3.13) we get

$$
\begin{aligned}
V(x)= & \operatorname{Res}_{s=1}\left(4 \zeta^{-1}(2 s) \zeta(s) L(s, \chi) x^{s} s^{-1}\right) \\
& +\sum_{m \leq y} \mu(m) E\left(\frac{x}{m^{2}}\right)+O\left(y^{-1 / 2} x^{1 / 2+\varepsilon}\right) \\
= & \frac{6}{\pi} x+\sum_{m \leq y} \mu(m) E\left(\frac{x}{m^{2}}\right)+O\left(y^{-1 / 2} x^{1 / 2+\varepsilon}\right) .
\end{aligned}
$$

Now we obtain the main result of this section.

Proposition 2. If $R H$ is true, then for $x^{\varepsilon} \leq y \leq x^{1 / 2-\varepsilon}$, we have

$$
E_{P}(x)=\sum_{m \leq y} \mu(m) E\left(\frac{x}{m^{2}}\right)+O\left(y^{-1 / 2} x^{1 / 2+\varepsilon}\right) .
$$

4. Proof of the Theorem. Take $y=x^{4 / 15}$ in Proposition 2. We only need to estimate the sum

$$
\sum_{m \sim M} \mu(m) E\left(\frac{x}{m^{2}}\right)
$$

for $x^{1 / 10} \ll M \ll y$. For $M \ll x^{1 / 10}$, we have trivially

$$
\sum_{m \leq x^{1 / 10}} \mu(m) E\left(\frac{x}{m^{2}}\right) \ll \sum_{m \leq x^{1 / 10}} x^{1 / 3} m^{-2 / 3} \ll x^{11 / 30} .
$$

By the well-known Voronoi formula of $E(t)$ (see [7], 13.8) we get 


$$
\begin{aligned}
x^{-\varepsilon} \sum_{m \sim M} \mu(m) & E\left(\frac{x}{m^{2}}\right) \\
& \ll\left|\sum_{m \sim M} \frac{\mu(m) x^{1 / 4}}{m^{1 / 2}} \sum_{n \leq x^{4 / 15}} \frac{r(n)}{n^{3 / 4}} e\left(\frac{\sqrt{n x}}{m}\right)\right|+x^{11 / 30} .
\end{aligned}
$$

It now suffices to show that $(1 \ll N \ll Y)$

$$
S(M, N)=\sum_{m \sim M} \frac{\mu(m) x^{1 / 4}}{m^{1 / 2}} \sum_{n \sim N} \frac{r(n)}{n^{3 / 4}} e\left(\frac{\sqrt{n x}}{m}\right) \ll x^{11 / 30+\varepsilon} .
$$

We consider three cases.

CASe $1: M \leq N \leq x^{4 / 15}$. Let

$$
T(M, N)=\sum_{m \sim M} \frac{\mu(m) M^{1 / 2}}{m^{1 / 2}} \sum_{n \sim N} \frac{r(n) N^{3 / 4-\varepsilon}}{n^{3 / 4}} e\left(\frac{\sqrt{n x}}{m}\right) .
$$

By Proposition 1 (take $(X, Y)=(N, M))$ we get

$$
\begin{aligned}
x^{-\varepsilon} T(M, N) \ll & N M^{1 / 2}+x^{2 / 20} N^{15 / 20} M^{11 / 20} \\
& +x^{2 / 23} N^{17 / 23} M^{14 / 23}+x^{1 / 12} N^{15 / 20} M^{11 / 18} \\
& +x^{2 / 20} N^{14 / 20} M^{12 / 20}+x^{1 / 20} N^{17 / 20} M^{12 / 20}
\end{aligned}
$$

whence

$$
\begin{aligned}
x^{-\varepsilon} S(M, N) \ll & x^{1 / 4} N^{1 / 4}+x^{7 / 20} M^{1 / 20}+x^{31 / 92} M^{9 / 92} \\
& +x^{1 / 3} M^{1 / 9}+x^{3 / 10} N^{1 / 10} M^{1 / 10} \\
\ll & x^{109 / 300} \ll x^{11 / 30} .
\end{aligned}
$$

CASE 2: $M^{1 / 4} \leq N \leq M$. We again use Proposition 1 to bound $S(M, N)$ (now take $(X, Y)=(M, N)$ ) and get

$$
\begin{aligned}
x^{-\varepsilon} S(M, N) \ll & x^{7 / 20} N^{1 / 10} M^{-1 / 20}+x^{31 / 92} N^{11 / 92} M^{-1 / 46} \\
& +x^{1 / 3} N^{1 / 9}+x^{7 / 20} N^{3 / 20} M^{-1 / 10}+x^{3 / 10} M^{1 / 5} \\
& +x^{1 / 4} N^{-1 / 4} M^{1 / 2} \\
\ll & x^{109 / 300}+x^{1 / 4} M^{7 / 16} \ll x^{11 / 30} .
\end{aligned}
$$

CASE 3: $N<M^{1 / 4}$. We use Lemma 10 to bound $T(M, N)$ and get

$$
x^{-\varepsilon} T(M, N) \ll x^{1 / 12} N^{11 / 12} M^{1 / 12}+N^{1 / 2} M^{11 / 12},
$$

whence we have

$$
\begin{aligned}
x^{-\varepsilon} S(M, N) \ll & x^{1 / 3} N^{1 / 6} M^{1 / 12}+x^{1 / 4} M^{5 / 12} \\
& +x^{1 / 3} M^{1 / 8}+x^{1 / 4} M^{5 / 12} \\
\ll & x^{11 / 30} .
\end{aligned}
$$

This completes the proof of the Theorem. 
Acknowledgements. The authors thank the referee for his or her kind and valuable suggestions.

\section{References}

[1] R. C. Baker and G. Harman, Numbers with a large prime factor, Acta Arith. 73 (1995), 119-145.

[2] E. Bombieri and H. Iwaniec, On the order of $\zeta\left(\frac{1}{2}+i t\right)$, Ann. Scuola Norm. Sup. Pisa 13 (1986), 449-472.

[3] E. Fouvry and H. Iw aniec, Exponential sums with monomials, J. Number Theory 33 (1989), 311-333.

[4] D. R. Heath-Brown, The Pjatecki-Šapiro prime number theorem, ibid. 16 (1983), $242-266$.

[5] D. Hensley, The number of lattice points within a contour and visible from the origin, Pacific J. Math. 166 (1994), 295-304.

[6] M. N. Huxley, Exponential sums and lattice points II, Proc. London Math. Soc. 66 (1993), 279-301.

[7] A. Ivić, The Riemann Zeta-function, Wiley, 1985.

[8] C. H. Jia, On the distribution of squarefree numbers (II), Sci. China Ser. A 8 (1992), $812-827$

[9] E. Krätzel, Lattice Points, Deutsch. Verlag Wiss., Berlin, 1988.

[10] S. H. Min, Methods of Number Theory, Science Press, Beijing, 1983 (in Chinese).

[11] W. G. Nowak, Primitive lattice points in rational ellipses and related arithmetical functions, Monatsh. Math. 106 (1988), 57-63.

[12] B. R. Srinivasan, The lattice point problem of many-dimensional hyperboloids II, Acta Arith. 8 (1963), 173-204.

Department of Mathematics

Shandong Normal University

Jinan, 250014, Shandong

P.R. China

E-mail: wgzhai@jn-public.sd.cninfo.net
Beijing Institute of Petrochemical Technology Daxing, Beijing 102600 P.R. China E-mail: biptiao@info.ind.cn.net 